\title{
Carcinoma of the cervix - the hopeless disease
}

To the Editor: Early in May, a friend in her late 70s here in KZN needed investigation and assessment for an abdominal cancer. She was able to access care in the private sector, and had a CT scan within 4 days; the appropriate management plan was begun in 7 days.

During the same week, the home-based carers in our small hospice found a 39-year-old mother of 4 children who was in great pain at home. She was on HAART, and had been diagnosed with cancer of the cervix 7 months previously in the district hospital. She was assessed by the Oncology Unit in our tertiary hospital 4 weeks later, and put on the waiting list for radiotherapy. There is a bottleneck in planning therapy because of the lack of CT scanning facilities. Her booking for CT scan and radiotherapy was in July - 9 months after diagnosis. She has a very aggressive tumour, and now has stage 4 disease with an upper segment deep vein thrombosis of her right leg, and serious urinary symptoms. Pain management is inadequate. She is set for a miserable death. A member of the Oncology Unit said they often have patients who progress from stage 2 to stage 4 disease before therapy can be commenced.

The fact that women with HIV on HAART are now living longer seems to be contributing to a rapid escalation in the number of women with carcinoma of the cervix coming from the Natal 
Midlands. Good motivations have been forwarded to the Department of Health for the Oncology Unit to be given its own CT scanner; but there has been no response yet, although this is an NHI Pilot Project District.

The situation has other spin-offs. The same week, we referred a patient who had just started HAART to the district hospital with a Pap smear and clinical findings of cancer of the cervix. It seems that the staff in the gynae clinic are so discouraged by the above scenarios that she was shunted to the end of the line, and asked to return for her biopsy to be done on two separate occasions. She has decided not to attend again. We have often observed healthcare providers behaving like this towards people with HIV before HAART became available. The dynamic seems to be a feeling of helplessness, in which the duty of caring seems to be lost.

The story also illustrates, on the one hand, how great is the need for NHI to be developed urgently. And on the other hand it underlines how very much must be achieved if the NHI is to provide a service that will be acceptable to South Africans who are used to private sector care. It illustrates too how important it is to teach that one can never say, 'There is nothing we can do for you.' - that the duty to care is as important as the duty to try to cure.

\section{J V Larsen}

Howick, South Africa

jon.larsen@iuncapped.co.za

S Afr Med J 2013;103(8):498-499. DOI:10.7196/SAMJ.7055 